\title{
Some Observations Of Highway Traffic In Long Queues
}

Karen R. Smilowitz, Carlos F. Daganzo, Michael J. Cassidy and Robert L. Bertini

416 McLaughlin Hall

Department of Civil and Environmental Engineering, and

Institute of Transportation Studies

University of California, Berkeley, CA 94720, USA

\begin{abstract}
The arrival times of vehicles traveling southbound along a two-lane, bi-directional highway were recorded at eight neighboring locations upstream of a bottleneck caused by an oversaturated traffic signal. Cumulative curves constructed from these observations describe completely and in great detail the evolution of the resulting long queues. These queues formed directly upstream of the signal when the signal's service rate fell below the southbound arrival rates, and never formed away from the bottleneck. The predictability of bottlenecks like the one studied here can be exploited to manage traffic more effectively.

The behavior of vehicles within the queue, however, was rather interesting. While the flow oscillations generated by the traffic signal were damped-out within one-half mile of the bottleneck, it was found that other oscillations arose within the queue farther upstream, at varied locations, and then grew in amplitude as they propagated in the upstream direction. Thus, the queue appeared to be stable close to the bottleneck and unstable far away. Oscillations never propagated beyond the upstream end of the queue, however; i.e., the unusual phenomena always arose after the onset of queuing and remained confined within the queue.

Some of these findings run contrary to current theories of traffic flow. As the data set collected in this study is unprecedented in scope and detail, and so that it may be of use to other researchers, it has been posted on the internet and is fully described here.
\end{abstract}

\section{KEY WORDS}

Bottleneck Observation; Car Following; Jam Development; Queueing; Traffic Instability 


\section{INTRODUCTION}

A better understanding of how queues form and propagate can lead to improved methods of managing highway traffic. Queue lengths and traffic spill-overs, for example, depend upon the spacing that drivers select in dense traffic and this points to the important role that car following theories can play in devising queue containment strategies. Although a number of such theories exist, the empirical evidence is scarce. Consequently, uncertainty surrounds even the most fundamental of issues. Especially telling in this regard is the lack of consensus as to why congestion (i.e., queues) arise (1); this is notable in that schemes for managing queues should stem from an understanding of their causes.

The study described here is part of an ongoing effort to identify the important and reproducible features of evolving traffic. The data collected to this end have been posted on the World Wide Web. They describe queues that formed immediately upstream of a traffic signal, propagated several miles further upstream and eventually began to dissipate toward the end of the rush. Analysis reveals that the bottleneck pulses were damped out before propagating through the entire queue and that other disturbances arose spontaneously at locations within the queue further upstream. These disturbances never propagated beyond the upstream end of the queue.

The findings augment the observations of a few previous studies. Some of these earlier works, along with an overview of the present study, are summarized in the following section. The experiment and the resulting data are described in sections 3 and 4, respectively. Section 5 presents the main findings from the analysis and their rationale. The sixth and final section presents some conclusions and suggestions for further work.

\section{BACKGROUND}

Some of the earliest car following studies took place on test tracks $(2,3)$. Although these efforts produced a wealth of very detailed observations, their realism is somewhat questionable since one cannot be sure that test track data really describe the behavior of drivers in highway traffic.

As an extension of these earlier efforts, researchers have more recently selected vehicles at random in real traffic and followed them in instrumented cars (4). These experiments, however, were not double-blind in that the subject drivers knew they were part of an experiment. Thus, it seems to us that these studies are also inconclusive.

Data taken in real traffic settings, when drivers are unaware of their participation in an experiment, can complement the above studies and shed additional light on the issues. Limited amounts of such data have been collected in past studies. In one widely-cited example, researchers constructed time-space vehicle trajectories by comparing vehicle positions on consecutive aerial photographs (5). These trajectories revealed much detail, but the data were limited both in the observation duration and the length of roadway examined. Video imaging methods have also been used as a (less laborious) means of extracting trajectories for longer periods of time (6). These methods, however, are still only capable of tracking vehicles over short distances.

In other studies, researchers have traced propagating disturbances over long sections of freeway using vehicle speeds and flows measured by loop detectors (7). This approach can yield much useful information, but it does not identify vehicles or their 
accumulation between detectors. Without these critical data, the results can be open to varying interpretation (1).

Fortunately, it has been recently demonstrated (8) that by working with cumulative curves of vehicle arrival number versus time, $\mathrm{N}$-curves, it is possible to extract individual vehicle information from loop detector data, provided that the road segments have simple geometries. The special insights derived from $\mathrm{N}$-curves have been known to the transportation profession for years (9). Unfortunately, loop detectors are often located near freeway entrance and exit ramps, which makes it difficult to separate the effects of lane changing, merging and diverging that occur at these access points from the pure car following behavior that determines the lengths of queues.

Our approach was to find a location without the above-mentioned complexities and use the best available data collection techniques for the chosen location. The highway segment selected (a very simple two-lane, bi-directional highway) was ideally suited for our purposes because it had a downstream traffic signal that generated a bottleneck when vehicle arrival rates rose during the morning rush and because there was very little vehicle overtaking and almost no side traffic.

Since the site chosen was not instrumented with loop detectors, human observers equipped with laptop computers were deployed to record the arrival times (and classes) of all southbound vehicles at eight observation points on two separate mornings. The $\mathrm{N}$ curves constructed from these data show the evolution of very long queues in a single traffic stream, without the complications that arise from vehicle lane changing, merging and/or diverging. In particular, it was possible to track the disturbances that propagated within these queues over their entire lives, to their final dissipations. The relatively small measurement errors incurred by using human observers are described in a later section. From our experience with experimental data, we believe our procedure of manual data collection to be comparable in accuracy with loop detectors.

\section{THE EXPERIMENT}

This section presents a thorough description of the site chosen for the study and of the methods used to extract the data. As regards the former, the data were collected on the four-mile segment of southbound San Pablo Dam Road shown in Figure 1. The site, located immediately north of the intersection with Wildcat Canyon Road, is a two-lane rural highway connecting the cities of Richmond and Orinda, California. The highway serves as a commuter alternative to a congested regional freeway. It has a posted speed limit of $50 \mathrm{mph}$ (commensurate with its design standards), some gentle horizontal and vertical curves, and a slight uphill grade (for southbound traffic) near the intersection with Wildcat Canyon Road. Adequate shoulders exist throughout the segment.

The near-absence of access and egress points and the minimal overtaking maneuvers at the site mean that vehicles maintained (approximately) their relative positions in the traffic stream; the amount of traffic that used the two access points at the San Pablo Dam reservoir was negligible and only a few vehicles, usually motorcycles, bypassed queues by driving on the shoulder. The downstream intersection at Wildcat Canyon Road is controlled by a vehicle-actuated traffic signal, which causes a queue of southbound vehicles to grow steadily during much of the morning rush. 
To collect the data, the eight observers were stationed along the site, as shown in Figure 1. While obscured from the view of drivers, these observers used laptop computers to record the arrival times of individual vehicles at their respective observation points. The laptop computers' internal clocks were synchronized prior to the first day of observation and a computer program was coded to append time values to keystrokes. The observers recorded each vehicle's arrival time and the vehicle class by pressing a specified key (i.e., "A" for automobile and "T" for commercial truck). The data format is explained in the following section.

Figure 1 shows that at the downstream end of the site, the observers were stationed in close proximity to one another. This arrangement yielded a higher measurement resolution for viewing disturbances shortly after they emanated from the traffic signal where we expected them to be growing. To ensure a complete study of the queue, the upstream-most observer was stationed four miles from the signal since it was estimated that the effects of the queue would not be felt beyond this location. All distances shown in Figure 1 were carefully identified with a measuring wheel.

A pilot car, which was easily distinguished from other vehicles (in that it had a bicycle mounted on its roof rack) cycled through the site during the study periods. When traveling southbound, its arrival times at each observation point were recorded (i.e., the observers pressed "B" on their computer keyboards). To provide some redundancy, the driver of the pilot car recorded the approximate times he passed each of the eight observation points while traveling southbound. On each of the two observation days, the beginning and ending of the study period were marked by the first and last passages of the pilot car. Furthermore, the intermediate arrival times of the pilot car were useful in prorating any (small) measurement errors made by the observers, as described in the following section. A second car cycled through the site to provide the observers with any needed assistance; e.g., to furnish observers with "replacement" computers when batteries had discharged.

Observations were collected from 6:45 a.m. to 9:00 a.m. on Tuesday, November 18 and again on Thursday, November 20, 1997. There was no precipitation on either day and visibility on the road was good.

\section{THE DATA}

The data from this study have been posted on the World Wide Web (at http://www.ce.berkeley.edu/ daganzo/spdr.html) and are available in two forms: "raw" data, which have not been altered, and "final" data, which have been filtered in an effort to correct for measurement errors. This section provides a full description of these data, including our filtering processes.

The raw data are contained in a total of sixteen text files, with each file holding the measurements made by a single observer on one of the two observation days. For example, file 1_A.txt holds the measurements of the upstream-most observer (i.e., observer 1) on the first day and file 8_B.txt the measurements of the downstream-most observer on day 2. As an illustration, a small portion of data file 5_A.txt is shown in Figure 2. The figure shows that each vehicle arrival time is recorded in hours, minutes, 
seconds and hundredths of seconds and is presented along with the vehicle's arrival number and class.

The comment "Computer Failure" shown in Figure 2 is used to flag one particular type of measurement error. Measurement errors came from several sources and they are described below so that other researchers may use the raw data with their own filtering processes. From the following discussion, however, it should become apparent that, given the nature of the measurement errors, all reasonable filtering processes would yield practically indistinguishable results. It should also be clear that these errors did not erode the integrity of the final data in any substantial way; i.e., despite the errors described below, the data are arguably the most detailed and accurate of their kind.

Hardware Malfunctions. In a few instances, records were apparently not entered by a human observer, but were due instead to some type of computer malfunction. These entries were easily identified because they gave rise to unduly small vehicle headways and they were often accompanied by a rectangular symbol, rather than an "A", "B", or "T", in the field designated for vehicle class. The raw data files include comment statements to flag each of these errant records and they have been purged from the final data.

Clock Synchronization Problems. Measurement errors also arose because the laptop computer internal clocks were not always well synchronized. The clocks in two of the laptops used on the first day of observation ran ahead of their counterparts by exactly one minute. This was made immediately evident by comparing the roadside data recorded with these two computers with the corresponding passage times measured (approximately) by the driver of the pilot car. The asynchronization was attributed to human error in setting the internal clocks and, in the final data set, the vehicle arrival times measured with these two computers were adjusted (i.e., reduced) by one minute. These adjustments are summarized in Table 1a.

Regrettably, the computers' clocks were not re-synchronized immediately prior to the second day of observation. As a consequence, by day 2, the clocks of all the computers had drifted by varying amounts. To salvage the second day's data, its vehicle arrival times taken at observation points 2 through 8 were adjusted as explained below.

First, the average free-flow vehicle trip times between each pair of contiguous observation points were estimated for day 1 . These estimates were made using the arrival times for the first 25 vehicles observed on that day, since these vehicles did not encounter residual queuing at the downstream intersection. The average travel times and standard errors of the sample means are presented in Table 1b. Notably, the standard error for each of these (seven) sample means never exceeded 1.2 seconds. The same calculations were then repeated for day 2, with the results shown in Table 1c. The large discrepancies between the measured averages on both days were attributed to synchronization error.

Thus, clock corrections that eliminated all the discrepancies between the recorded free-flow trip times on both days were chosen. (If no correction is assigned to observer 1, then the clock correction for location $\mathrm{j}>1$ is simply the difference of the average trip times from observer 1 to observer $\mathrm{j}$ on both days.) These corrections are shown in Table 1d. This adjustment method was deemed to be more reliable than using the approximate trip times measured on day 2 by the driver of the pilot car.

Computer Failure. Figure 2 shows a gap in the observations that extended for almost four minutes and is labeled "Computer Failure." On occasion, a computer required 
replacement (while in the field) because its battery had fully discharged. The few replacements that resulted in the loss of recorded observations are flagged in the raw data as a "Computer Failure."

To address the resulting loss of information, the observation days were partitioned into smaller mini-datasets consisting of all the vehicular information collected between consecutive passages of the southbound pilot car. Ideally, each of the eight observers would measure the same number of vehicles in each mini-dataset. This was approximately the case, except when a computer failure created large gaps in the recorded observations. Therefore, measurements taken (at the observation point) subsequent to a computer failure were removed from the mini-dataset if the gap in the records exceeded one minute. In total, this truncation was performed only on two occasions, at two different observation points. In one of these occasions, which occurred during the penultimate mini-dataset of day 2, the replacement computer could not be restarted in time to be useful for the last min-dataset. Two counts were also truncated for the last mini-dataset of day 2 because observations at these locations were terminated shortly before the final passage of the southbound pilot car.

Human Error. All other (small) discrepancies between the vehicle counts in a mini-dataset were attributed to human error, such as a missed observation or an unwarranted keystroke. The counting errors created by small glitches in computer exchanges were likewise placed in this category. Since these errors could not be individually identified, they were prorated equally over each mini-dataset by renormalizing the counts; i.e., each vehicle's arrival number, as recorded by a jth observer, was multiplied by the ratio $\bar{N} / \mathrm{N}^{(j)}$, where $\mathrm{N}^{(\mathrm{i})}$ is the number of vehicles counted by observer $\mathrm{j}$ in the mini-dataset and $\overline{\mathrm{N}}$ is the average of the $\mathrm{N}^{(\mathrm{j})}$ across observers. Of note, the four truncated counts discussed under "Computer Failure" were not adjusted and were not included in the computation of an $\overline{\mathrm{N}}$.

On the second day, observer 5 failed to record the second passage of the pilot car. It was assumed that the observer inadvertently logged this arrival by selecting an A, as if the pilot car were a passenger car. A record of vehicle class was subsequently changed from an A to a B. This record was chosen in the maximum likelihood way so as to yield the same ratio of vehicle counts in the first and second mini-runs as was typical of the other observers. The standard error in this procedure should be on the order of 1 or 2 vehicles.

Filtered in the above way, the final data were stored in two Excel spreadsheets named D1Final.xls and D2Final.xls, for days 1 and 2, respectively. For illustrative purposes, a portion of file D1Final.xls is shown in Figure 3. The following section presents some evaluation of these data and the findings that resulted. It will become apparent from the figures presented in the next sections that the changes made by the above filtering process are very minor. 


\section{SOME ANALYSIS}

Figure 4 shows the conventional curves of vehicle count versus time, $N(j, t)$, for day 2, where

$N(j, t)=$ the (filtered) cumulative number of vehicles to pass stationary observer $\mathrm{j}$ by time $\mathrm{t}$, measured from the first passage of the southbound pilot car, $\mathrm{j}=$ $1,2, \ldots, 8$.

Figure 4 indicates that the flow of southbound vehicles past $j=8$ began to drop at around 7:04 a.m. -- the reader may use a straight edge to verify the change in the trend of $N(8, t)$ around this time. We believe that this drop in flow was caused by growth in the conflicting traffic streams at Wildcat Canyon Road, because the stop-and-go features of $N(8, t)$ reveal that the drop is due exclusively to an increase in the duration and frequency of the episodes with zero flow (when "red" was displayed to southbound traffic) and not to any significant change in the slope of $N(8, t)$ during the "green".

Shortly following the overall flow reduction at $j=8$, the slopes of some of the upstream $\mathrm{N}$-curves dropped in sequence. Figure 4 includes labels indicating when these events took place at the various observation points. Note that the drop in slope at an observation point $j$ is simply the flow reduction brought about by the growing queue. As one would expect, after the drop at $j$, when the queue has grown beyond this location, the vehicle trip times (i.e., the horizontal displacements) and the accumulations (i.e., the vertical displacements) between observation points $j-1$ and $j$ are no longer minimum. Note that the queue never propagated to $j=2$ and thus, vehicles arrived at $j=2$ without delay. This is evident because the horizontal displacements between the curves at $j=1$ and $j=2$ did not noticeably increase from what is displayed at the beginning of the observation period.

Downstream curve $N(8, t)$ continued to drop gradually until about 7:30 a.m., owing to gradual growth in the conflicting traffic streams at Wildcat Canyon Road. Quite apart from this effect, a final flow reduction occurred at $j=8$ when, at about 8:27 a.m., a queue from further downstream spilled-over. This is evidenced by the reduced saturation flows at Wildcat Canyon Road during the "green" periods, which are clearly visible in curve $N(8, t)$.

The slope of upstream-most $N(1, t)$ dropped at about 7:23. It is clear that this slope change was due to a reduction in vehicle arrivals from further upstream because curve $N(2, t)$ adopted the same slope (approximately) one trip time later. Similarly linked changes in the arrival rates at observation points 1 and 2 are visible from $N(1, t)$ and $N(2, t)$ at later times. Note in particular how the wiggles in $N(1, t)$ are passed horizontally to $N(2, t)$. This is an additional indication of the absence of queuing. (10)

The inset of Figure 4 shows that the pronounced stop-and-go patterns seen at $j=8$ were damped-out before reaching $j=6$. Note how much smoother curve $N(6, t)$ is than curve $N(8, t)$. Remarkably, however, new disturbances appeared further upstream. In some instances, upstream traffic flow was reduced to zero as it completely jammed and a few such examples are labeled in the inset. These jams propagated upstream and always died upon (or before) reaching the upstream end of the queue. Oscillations in flow near the tail end of the queue appeared to be softer, as evidenced by curve $N(3, t)$, which exhibits a pattern of gradual slope changes. The observed fluctuations in flow never affected traffic upstream of the queue. There was no evidence that instabilities or jams 
occurred in freely flowing traffic well upstream of the signal. The latter should not be surprising; if flow collapses occur at all (and we are not certain that they do), they would not likely appear in a situation with a maximum flow of 1500 vehicles/hour. The interested reader may refer to (1) for more discussion of this issue.

Figure 5 shows the curves constructed with the final data from day 1. Study of these curves reveals a queue evolution similar to what was observed on day 2. Notable differences are that the queue on day 1 appears to reach observer 2 and to have dissipated more fully by the end of the observation period.

\section{CONCLUSIONS}

This paper has described a study of queue evolution at a highway bottleneck. The sources of measurement error in the data collected here were explained in section 3 . The few large errors that arose (e.g., due to failed battery exchanges) could be easily identified and corrected, thanks to the redundancy built into the experiment. The remaining errors were so small that they could be distributed evenly across each mini-data-set with insignificant changes. As an illustration of this, Figure 6 presents the unadjusted $N$-curves for both observation days. The reader is invited to compare these curves with their counterparts in Figures 4 and 5, and to note the negligible differences. We believe the accuracy of this experiment is comparable to experiments with loop detectors.

Visual inspection of the $N$-curves indicates that the queues formed in predictable ways at the most obvious inhomogeneity of this facility, the traffic signal. It was also found that the queues dissipated in predictable ways; e.g., when vehicle arrival rates from further upstream diminished and/or the bottleneck service rate increased.

There were some unusual and unexpected features in the data as well. For example, stop-and-go jams, uncorrelated with the traffic signal, were observed in queued traffic without passing. This is interesting because the absence of passing means that traffic information is unlikely to overtake vehicles, and that theories that include such possibility (kinetic theory, high order fluid models, etc.) do not explain what is being observed here. A further puzzle is that while instabilities seem to grow in amplitude far from the bottleneck, the pronounced stop-and-go oscillations of the bottleneck itself were rapidly damped-out within one-half mile of it.

Perhaps these unusual observations are unique to the site studied here. For example, the predictability of the server (i.e., the traffic signal), and/or the low risk of being overtaken, may have motivated drivers to follow downstream vehicles in unusually relaxed ways. We would very much hope that other researchers would attempt to replicate or disprove our observations at other sites. In any case, and even if stranger phenomena are observed at other sites, theories to predict these unusual features may not be needed. The usefulness of this research for practice will come from further research. From an engineering perspective, one is most interested in predicting approximately the time-dependent queue lengths (distances) due to control actions, and the ensuing delays. To do this acceptably well it suffices to predict the $N$-curves approximately at intermediate locations (e.g., given the curves at the highway's upstream and downstream boundaries) even if one cannot predict the detailed location of all the wiggles. This seems to have been the objective behind Newell's simplified theory of kinematic waves (11) and it may be the 
desirable form for future theories of highway traffic. We believe that these data and similar data sets obtained elsewhere can be valuable for testing practical theories that would predict $N$-curves.

\section{ACKNOWLEDGEMENTS}

The authors would like to thank Flavio Baita, Lyle DeVries, Reinaldo Garcia, Jamie Lawson, Raymond Lew, Michael Mauch, and Joseph Wanat for their help in collecting the data. Research supported in part by PATH MOU-305 to the University of California, Berkeley. 


\section{REFERENCES}

1. Daganzo, C.F., M.J. Cassidy and R.L.Bertini, Possible Explanations of Phase Transitions in Highway Traffic, to be published in Transportation Research.

2. Herman, R. and R. Rothery (1965) Propagation of Disturbances in Vehicular Platoons in (ed. L. Edie, R. Herman, and R. Rothery), Proceedings from the Third International Symposium on Traffic Theory, 14-25.

3. Franklin, R.E. (1965) Single-Lane Traffic Flow on Circular and Straight Tracks in (ed. L. Edie, R. Herman, and R. Rothery), Proceedings from the Third International Symposium on Traffic Theory, 42-55.

4. Bleile, T. (1997) A New Microscopic Model for Car-Following Behavior in Urban Traffic, as well as D. Manstetten, W. Krautter, and T. Schwab (1997) Traffic Simulation Supporting Urban Control Systems Development, in Proc. 4th World Congress in Intelligent Transport Systems, Berlin, 1997, and the analyses of these data in Helbing, D. and Tilch, B. "Generalized force model of traffic dynamics" (preprint.)

5. Treiterer, J. and J. Myers (1974) The Hysteresis Phenomenon in Traffic Flow in (ed. D.J. Buckley), Proceedings from the Sixth International Symposium on Transportation and Traffic Theory, 13-38.

6. Coifman, B. (1997) Time Space Diagrams for Thirteen Shock Waves, University of California, California PATH Working Paper, UCB-ITS-PWP-97-1.

7. Kerner, B.S., and H. Rehborn (1997) Experimental Properties of Phase Transitions in Traffic Flow. Physics Review Letters, 79, No. 20, 4030 -4033.

8. Cassidy, M.J. and J.R. Windover (1998) Driver Memory: Motorists Selection and Retention of Individualized Headways in Highway Traffic. Transportation Research, 32A, No 2, 129-137.

9. Makigami, Y., G.F. Newell, and R. Rothery (1971) Three Dimensional Representation of Traffic Flow. Transportation Science, Vol. 5, No. 3, pp. 302313.

10. Cassidy, M. and J. Windover (1995) Methodology for Assessing Dynamics of Freeway Traffic Flow", Trans. Res. Rec. 1484, 73-79.

11. Newell, G.F. (1993) A Simplified Theory of Kinematic Waves in Highway Traffic, Part I: General Theory. Transportation Research. 27B, 281-287. 


\section{LIST OF TABLES}

Table 1a. Clock Synchronization Corrections, Day 1

1b. Free Flow Travel Times, Day 1

1c. Free Flow Travel Times, Day 2

1d. Clock Synchronization Corrections, Day 2

\section{LIST OF FIGURES}

Figure 1. Experiment Site

Figure 2. $\quad$ Sample from 5_A: Original Vehicle Count Files (Uncorrected)

Figure 3. Sample from D1Final: Final Vehicle Count Files (Corrected)

Figure 4. $\quad$ N-Curves, (Adjusted) Day 1

Figure 5. N-Curves, (Adjusted) Day 2

Figure 6. N-Curves (Unadjusted) Days 1 and 2 
Table 1a: Clock Synchronization Corrections (min:sec)

Tuesday, November 18, 1997 (Day 1)

\begin{tabular}{|c|l|}
\hline Observer & Change \\
\hline 2 & $01: 00$ subtracted from all measured arrival times \\
3 & $01: 00$ subtracted from all arrival times measured between $6: 44: 33$ and $8: 42: 14: 82$ \\
& (the period when a computer with an asynchronized clock was used by observer 3) \\
\hline
\end{tabular}

Table 1b: Free Flow Travel Times (min:sec)

Tuesday, November 18, 1997 (Day 1)

\begin{tabular}{|c|c|c|c|}
\hline Segment & $\begin{array}{l}\text { Estimated Average Free } \\
\text { Flow Travel Time }\end{array}$ & $\begin{array}{l}\text { Standard Deviation of the } \\
\text { Population }\end{array}$ & $\begin{array}{l}\text { Standard Error of the } \\
\text { Sample Mean }\end{array}$ \\
\hline $1-2$ & $02: 16.6$ & $00: 03.7$ & $00: 00.7$ \\
$2-3$ & $00: 30.3$ & $00: 01.6$ & $00: 00.3$ \\
$3-4$ & $00: 40.5$ & $00: 01.7$ & $00: 00.3$ \\
$4-5$ & $00: 15.2$ & $00: 02.5$ & $00: 00.5$ \\
$5-6$ & $00: 24.1$ & $00: 03.2$ & $00: 00.6$ \\
$6-7$ & $00: 36.2$ & $00: 06.1$ & $00: 01.2$ \\
$7-8$ & $00: 25.8$ & $00: 01.0$ & $00: 00.2$ \\
\hline
\end{tabular}

Table 1c: Free Flow Travel Times (min:sec)

Thursday, November 20, 1997 (Day 2)

\begin{tabular}{|c|c|c|c|}
\hline Segment & $\begin{array}{l}\text { Estimated Average Free } \\
\text { Flow Travel Time }\end{array}$ & $\begin{array}{l}\text { Standard Deviation of the } \\
\text { Population }\end{array}$ & $\begin{array}{l}\text { Standard Error of the } \\
\text { Sample Mean }\end{array}$ \\
\hline $1-2$ & $02: 26.3$ & $00: 02.1$ & $00: 00.4$ \\
$2-3$ & $00: 17.8$ & $00: 01.0$ & $00: 00.2$ \\
$3-4$ & $00: 45.3$ & $00: 01.9$ & $00: 00.4$ \\
$4-5$ & $00: 19.4$ & $00: 01.3$ & $00: 00.3$ \\
$5-6$ & $00: 11.2$ & $00: 01.8$ & $00: 00.4$ \\
$6-7$ & $00: 31.0$ & $00: 01.1$ & $00: 00.2$ \\
$7-8$ & $-00: 10.4$ & $00: 01.5$ & $00: 00.3$ \\
\hline
\end{tabular}

Table 1d: Clock Synchronization Corrections (min:sec)

Thursday, November 20, 1997 (Day 2)

\begin{tabular}{|c|l|}
\hline Observer & Change \\
\hline 2 & $01: 09.7$ subtracted from all measured arrival times \\
& (includes 1 minute correction for original asynchronization of the clock) \\
3 & $00: 02.8$ added to all measured arrival times \\
5 & $00: 02.0$ subtracted from all measured arrival times \\
6 & $00: 06.2$ subtracted from all measured arrival times \\
7 & $00: 06.8$ added to all measured arrival times \\
8 & $00: 12.0$ added to all measured arrival times \\
\hline
\end{tabular}




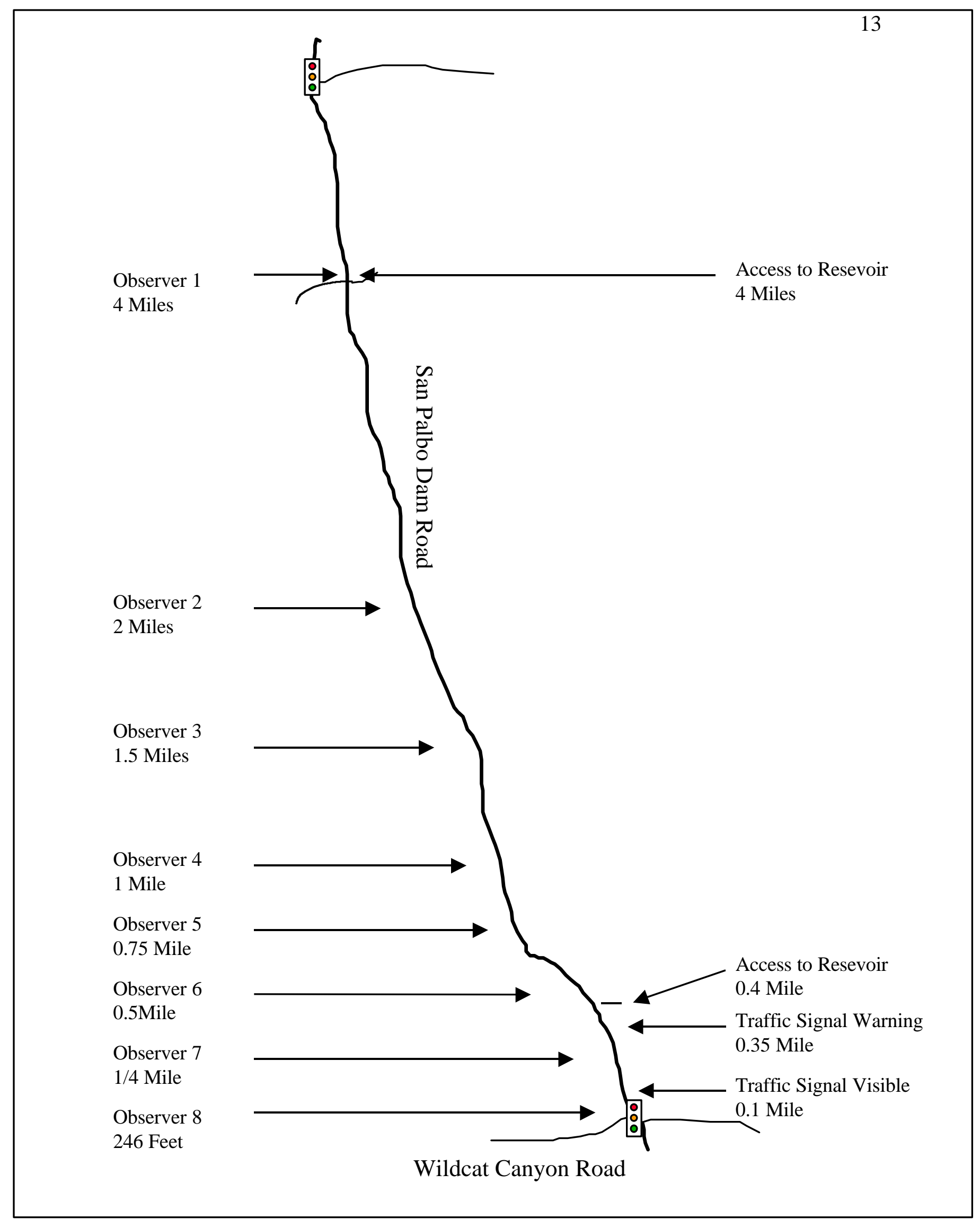

Figure 1: Experiment Site

Note: All distances measured from Wildcat Canyon Road 
Figure 2: Sample from File 5_A.txt

Location $\quad 15602$ feet from observer 1

Tuesday, November 18, 1997 (Day 1)

\begin{tabular}{|cccccc|}
\hline Hours & Minutes & Seconds & Hundredths & Vehicle \# & $\begin{array}{c}\text { Vehicle } \\
\text { Class }\end{array}$ \\
\hline 8 & 21 & 28 & 24 & 1678 & $\mathrm{~A}$ \\
8 & 21 & 29 & 99 & 1679 & $\mathrm{~A}$ \\
8 & 21 & 31 & 97 & 1680 & $\mathrm{~A}$ \\
8 & 21 & 35 & 43 & 1681 & $\mathrm{~A}$ \\
8 & 21 & 36 & 69 & 1682 & $\mathrm{~A}$ \\
8 & 21 & 37 & 74 & 1683 & $\mathrm{~A}$ \\
8 & 21 & 41 & 42 & 1684 & $\mathrm{~A}$ \\
8 & 21 & 43 & 83 & 1685 & $\mathrm{~A}$ \\
8 & 21 & 46 & 96 & 1686 & $\mathrm{~A}$ \\
8 & 21 & 50 & 37 & 1687 & $\mathrm{~A}$ \\
\hline Computer Failure & & & & & \\
\hline 8 & 25 & 40 & 12 & 1688 & $\mathrm{~A}$ \\
8 & 25 & 43 & 31 & 1689 & $\mathrm{~A}$ \\
8 & 25 & 46 & 38 & 1690 & $\mathrm{~A}$ \\
8 & 25 & 49 & 52 & 1691 & $\mathrm{~A}$ \\
8 & 25 & 55 & 72 & 1692 & $\mathrm{~A}$ \\
8 & 26 & 0 & 83 & 1693 & $\mathrm{~A}$ \\
8 & 26 & 2 & 92 & 1694 & $\mathrm{~A}$ \\
8 & 26 & 4 & 78 & 1695 & $\mathrm{~A}$ \\
8 & 26 & 8 & 46 & 1696 & $\mathrm{~A}$ \\
8 & 26 & 12 & 25 & 1697 & $\mathrm{~A}$ \\
\hline
\end{tabular}


Day 1
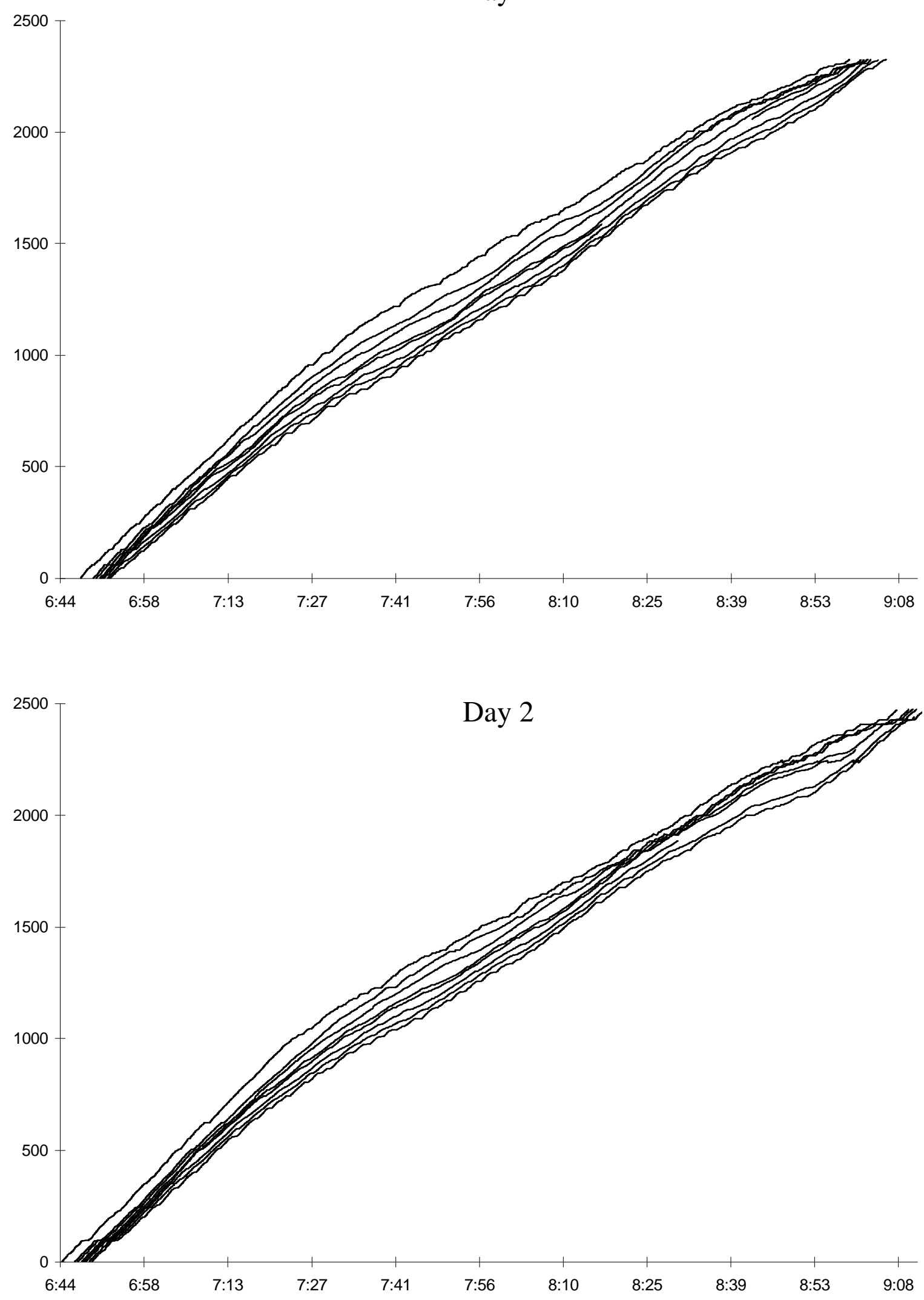

Figure 6: Unadjusted Curves of N(j,t,) 